\title{
Coupling Analysis of GaAs-Based Microdisk Lasers With Different External Claddings
}

\author{
Danyu Liu, Student Member, IEEE, Haroldo T. Hattori, Senior Member, IEEE, OSA, Lan Fu, Senior Member, IEEE, \\ Hark Hoe Tan, Senior Member, IEEE, and Chennupati Jagadish, Fellow, IEEE, Fellow, OSA
}

\begin{abstract}
In this paper, we analyze the coupling of light from a GaAs microdisk laser into a waveguide. Starting from an air cladding, we examine several configurations to couple light into the waveguide with different cladding structures aimed to foster light coupling into the waveguide: photonic crystal and metallic (plasmonic cladding). In these coupling schemes, we tried to optimize the coupling of the emitted light into the waveguide, while maintaining a reasonable quality factor to allow the lasing operation of the device. We show that a plasmonic layer, introduced beside the waveguide can lead to a significant improvement in the coupling efficiency, reaching an efficiency close to $80 \%$.
\end{abstract}

Index Terms-Microdisk lasers, photonic crystals, plasmonics, whispering-gallery modes.

\section{INTRODUCTION}

$\mathbf{E}$ LECTRONIC technology has been revolutionized by integrated circuits. The advent of integrated circuits has led to cheaper, more reliable, and more compact electronic devices and systems. Moreover, it has also allowed the insertion of many new functions in these systems. A typical device that strongly relies on electronic integration is the mobile telephone handset, which only became available with the advent of electronic integrated circuits.

Now, if optical circuits want to play a more important role in future, optical devices and systems need to achieve a much higher degree of integration. However, if we wish to produce optical devices that are very small and compact, it is necessary to confine light in small regions. There are three basic physical mechanisms to confine light in small spaces: the photonic bandgap effect [1]-[4], total internal reflection [5]-[20], and plasmonic propagation [21]-[24].

Among these techniques, total internal reflection-based devices are very popular due to their high performance and not so demanding fabrication. These devices generally employ polygonal resonators such as squares, triangles, and microdisks. Although triangular resonators have fewer modes than microdisk

Manuscript received December 04, 2008; revised June 15, 2009. First published July 21, 2009; current version published September 25, 2009. This work was supported by the Australian Research Council (ARC).

D. Liu, L. Fu, H. H. Tan, and C. Jagadish are with the Department of Electronic Materials Engineering, Research School of Physicals Science and Engineering, The Australian National University, Canberra 0200, Australia (e-mails: ldy109@ rsphysse.anu.edu.au, dliu020@gmail.com; fu1109@rsphysse.anu.edu.au; hoe.tan@anu.edu.au; c.jagadish@ieee.org).

H. T. Hattori is with the The University of New South Wales, The Australian Defence Force Academy, Canberra 2600, Australia (e-mail: H.Hattori@adfa. edu.au).

Digital Object Identifier 10.1109/JLT.2009.2028161 lasers, they generally have lower quality factors $(Q)$. The presence of high $Q$ resonances might be the main reason for microdisk resonators to be so popular.

The study of light coupling from polygonal resonators into waveguides has a long history. In general, light coming from (or sometimes entering into) a given polygon (e.g., square and corner-cut square [25], microdisk and microring [26], elliptical [27], racetrack [28] resonators) is evanescently coupled into a waveguide. This waveguide can be either positioned in the same plane of the core layer of the resonator [27] or in a different plane [16], [17]. Also, the waveguide can be a straight waveguide or wrapped around the cavities to improve the coupling efficiency [18], [19], [29].

In this article, we examine different ways to couple light from a microdisk laser into a waveguide. The main focus of this article is to compare different coupling schemes that could maximize the coupling of light into the waveguide. These coupling schemes are based on the introduction of different cladding structures to the laser and waveguide structures: low refractive index cladding (air), periodic cladding operating in its bandgap region [photonic crystal $(\mathrm{PhC})]$, and metallic (plasmonic) cladding. The basic idea is to increase the coupling rate into the waveguide when compared with other loss rates (e.g., radiation losses), so that it would be easier for light to be coupled into the waveguide, producing an overall increase in the coupling efficiency (into the waveguide).

However, there is a detrimental effect when the coupling rate into the waveguide is increased. Starting from an isolated microdisk, it is possible to find modes with very high-quality factors $\left(Q_{\mathrm{i}}\right)$. However, the introduction of a coupled waveguide leads to an increase of the overall losses in the cavity (more light is being (lost) coupled into the waveguide). The net effect is the reduction of this quality factor of the system to a new value $\left(Q_{1}<Q_{\mathrm{i}}\right)$, when the microdisk is loaded with a waveguide. If this loaded quality factor $Q_{1}$ falls below a certain value (e.g., $Q_{1}<600$ ), the threshold pump optical power would be so high that lasing would be virtually impossible. Hence, we need to make sure that not only the coupling of light into the waveguide is efficient but also that the quality factor of the resonant mode does not fall below a minimum value beyond where lasing would be impossible.

\section{General Description of the Devices and Analysis of STRUCTURES BASED UPON TOTAL INTERNAL REFLECTION}

The epitaxially layered structure that will be used to fabricate the laser devices is shown in Fig. 1. The core layer incorporates three 7.4-nm-thick $\mathrm{In}_{0.2} \mathrm{Ga}_{0.8}$ As quantum wells (QWs), 


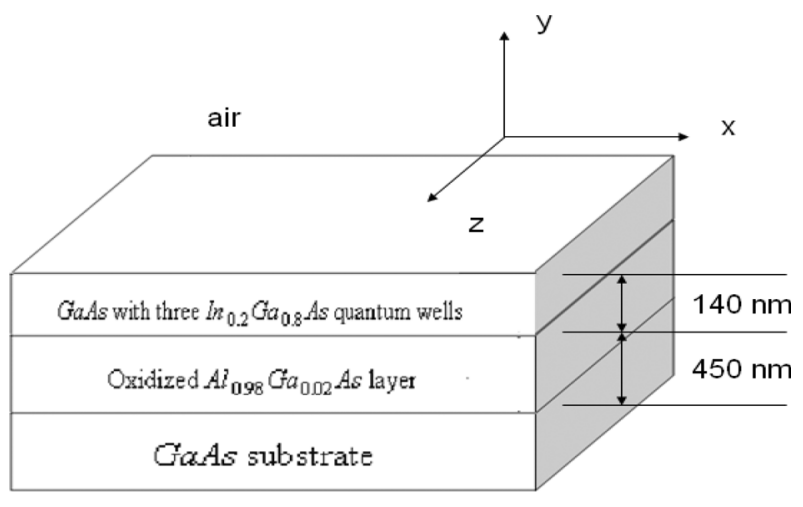

Fig. 1. Epitaxially layered structure of the optically pumped devices.

separated by $6 \mathrm{~nm}$ GaAs confinement barriers. The total thickness of this layer is $140 \mathrm{~nm}$. These QWs provide a maximum gain at $\lambda=980 \mathrm{~nm}$ ( $\lambda$ is the free-space wavelength) with a linewidth of about $40 \mathrm{~nm}$. In order to provide a good vertical confinement of light in the core region, a high aluminum content $\mathrm{Al}_{0.98} \mathrm{Ga}_{0.02} \mathrm{As}$ layer is grown below the core region. This layer will be wet oxidized later. The thickness of the $\mathrm{Al}_{0.98} \mathrm{Ga}_{0.02} \mathrm{As}$ layer is about $450 \mathrm{~nm}$ (before oxidation takes place). Both of these two layers are grown on a GaAs substrate by using metalorganic chemical vapor deposition system. This epitaxially layered structure is grown to fabricate devices that will work under optical pumping. This epitaxially layered structure is mainly designed to fabricate devices that operate under TE modes (main magnetic field in the $y$-direction) as shown in Fig. 1.

In order to analyze the performance of these devices, commercial 3-D FDTD software is used. A Gaussian source with spot size diameter of $300 \mathrm{~nm}$ is placed close to the edge of the microdisk structure. Power/magnetic field monitors are placed in the middle of the waveguide. The computation region is terminated by perfectly matched layers. The grid sizes in both $x$ and $z$ directions are $40 \mathrm{~nm}$ and $20 \mathrm{~nm}$ in the $y$ direction. The time step is $6.67 \times 10^{-17} \mathrm{~s}$.

Before considering different schemes of coupling the emitted light into the waveguide, let us first consider the microdisk alone. This is a well-known structure in which the confinement of light in the microcavity is achieved by total internal reflection. Total internal reflection occurs at the boundary between the high refractive index material (in our case GaAs) and the low refractive index material (air). This structure supports high-quality factor $(Q)$ whispering gallery resonant modes. The resonant frequencies of these TE-like WGMs $\mathrm{TE}_{\mathrm{m}, 1}(\mathrm{~m}$ and $\mathrm{l}$ are angular and radial mode numbers) can be determined by solving the following eigenvalue equation [20]:

$$
J_{\mathrm{m}}\left(n_{\mathrm{eff}} k R\right) H_{\mathrm{m}}^{\prime(2)}(\mathrm{KR})=\eta J_{\mathrm{m}}^{\prime}\left(n_{\mathrm{eff}} \mathrm{kR}\right) H_{\mathrm{m}}^{(2)}(K R)
$$

where $J_{\mathrm{m}}$ and $H_{\mathrm{m}}^{(2)}$ are the Bessel and second-kind Hankel functions of order $\mathrm{m}, k$ is the free-space wave number, $n_{\mathrm{eff}}$ is the effective index of the TE mode, and $\eta$ equals to $1 / n_{\text {eff }}$ for the TE-like WGMs [20]. By solving this equation, we find out that the main resonant mode (with the highest quality factor in the gain linewidth) is the $\mathrm{TE}_{18,2}$.

The first case to be considered here is a microdisk coupled to a "wrapped" waveguide [Fig. 2(a)]. This is a reference structure that will be used to compare the performance of different laser structures. The width of the waveguide is chosen as $500 \mathrm{~nm}$ and is a little bit larger than a single-mode waveguide, which should have a width of less than $350 \mathrm{~nm}$. In fact, this waveguide can support two transversal modes, but the higher order mode has much higher radiation losses than the dominant mode since its effective index is close to the refractive index of the oxide region. The air gap between the wrapped waveguide and the microdisk resonator has a width of $150 \mathrm{~nm}$. Although a stronger coupling would result with smaller gap widths, this value was chosen to facilitate the fabrication of future devices: gap widths larger than $100 \mathrm{~nm}$ could be easily fabricated with electron beam lithography/reactive ion etching systems and even with focused ion beam milling system.

In order to identify different resonant modes and assess their quality factors in the absence of a gain medium $(Q)$, we place a magnetic field monitor (field component $H_{\mathrm{y}}$, which is the strongest magnetic field component for TE modes) at the center of the waveguide. We then place a Gaussian source in the microdisk that has a finite duration in time (a pulse that decays quickly in time with a broadband spectrum) and detect the response of the system to this excitation. After processing this response, we obtain its frequency response by using fast Fourier transform algorithms. The magnetic field spectrum $\left(H_{\mathrm{y}}\right)$ allows us to determine the wavelength and quality factors of the resonant modes. Once we identify the desirable resonant peak (generally the peak with the highest $Q$ in the gain linewidth of the QWs, which is the first resonant mode to lase), we launch a continuous sinusoidal wave at this chosen wavelength. From laser theory [30], [31], all the power that is generated from a laser resonator must exit through the cavity "losses" to avoid accumulation of energy in the cavity. Based on this premise, we can place power detectors around the cavity to determine how much of the generated power is coupled into the waveguide, assessing the coupling efficiency (into the waveguide).

The magnetic field spectrum $\left(H_{\mathrm{y}}\right)$ for the structure in Fig. 2(a) obtained at the center of the waveguide is shown in Fig. 2(b). The main peak appears at $\lambda=972 \mathrm{~nm}$ with $Q=6500$. Its field distribution is shown in Fig. 2(c). A power budget analysis indicates that $61 \%$ of the power is coupled into the waveguide (there is more power being coupled into the upper waveguide because the source is generating a clockwise propagating wave). About $10 \%$ of the input power is lost downward in the oxide region, while $3 \%$ escapes through the air region. All the remaining power escapes in the lateral direction.

The coupling efficiency could be increased by reducing the air gap width to zero. This merged structure is similar to that presented in [19], but the waveguide is not placed at one of the edges of the microdisk laser. One immediate effect is a small shift in the resonant peak of the main mode to $\lambda=971 \mathrm{~nm}$. There is also a reduction of the quality factor of this peak to 4000 , which can be easily explained by an improved coupling of light from the microdisk structure into the merged waveguide. This reduction is not very drastic and still allows the device to lase ( $Q$ must be higher than 600 to allow lasing with our setup).

A power budget analysis at $\lambda=971 \mathrm{~nm}$ indicates that about $70 \%$ of the input power is now being coupled into the waveguide, an increase of $10 \%$ in the coupling efficiency with a 


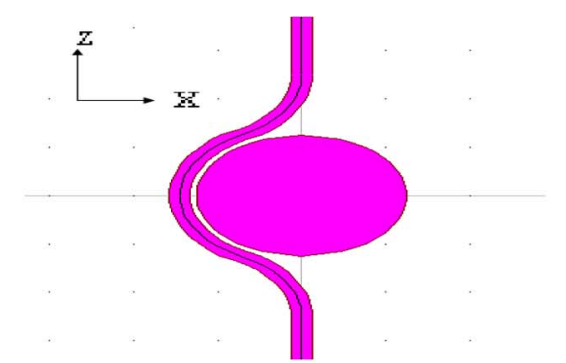

(a)

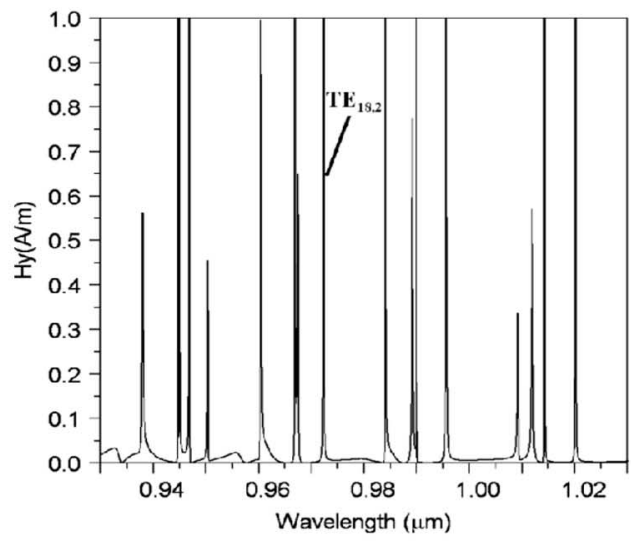

(b)

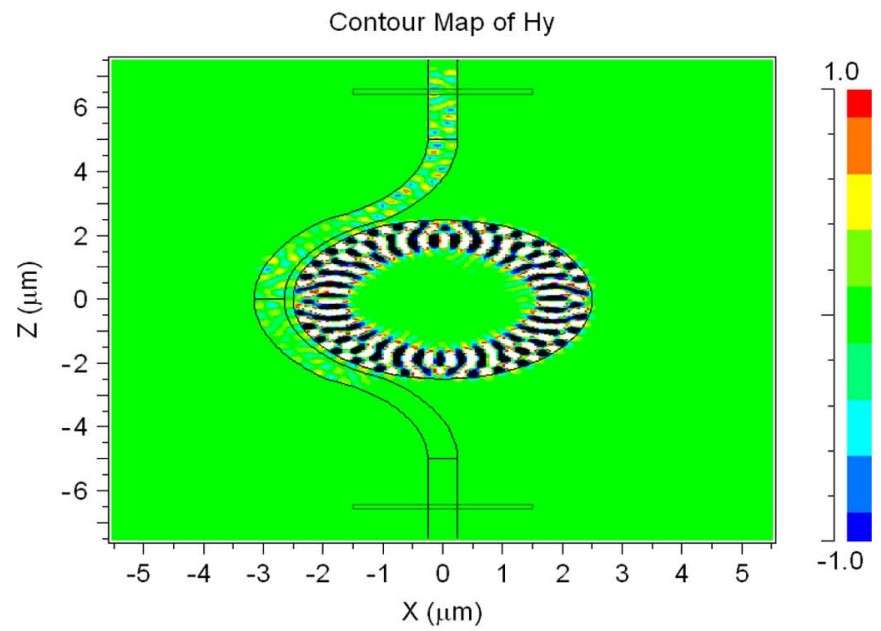

(c)

Fig. 2. (a) Top view of the microdisk laser coupled into a wrapped waveguide. (b) Magnetic field $\left(H_{\mathrm{y}}\right)$ spectrum at the center of the waveguide. (c) Magnetic field distribution $\left(H_{\mathrm{y}}\right)$ at the resonant wavelength of $972 \mathrm{~nm}$.

reduction of $Q$ of $40 \%$. It looks apparent that we could have removed the wrapped waveguide and substituted this complex waveguide by straight waveguides at the edge of the microdisk resonator, as was done in [9], [20]. This procedure does not work well because of the symmetry of the whispering gallery mode, leading to lower coupling efficiencies (lower than 50\%).

\section{Coupling of Light Assisted by a PhC Cladding}

Lateral radiation losses limit the coupling of light into the waveguide. In order to further increase the power coupling efficiency, we can use a PhC cladding as shown in Fig. 3. This PhC consists of a triangular lattice of air holes with lattice constant of $277 \mathrm{~nm}$ and radius of $100 \mathrm{~nm}$. The bandgap region of this

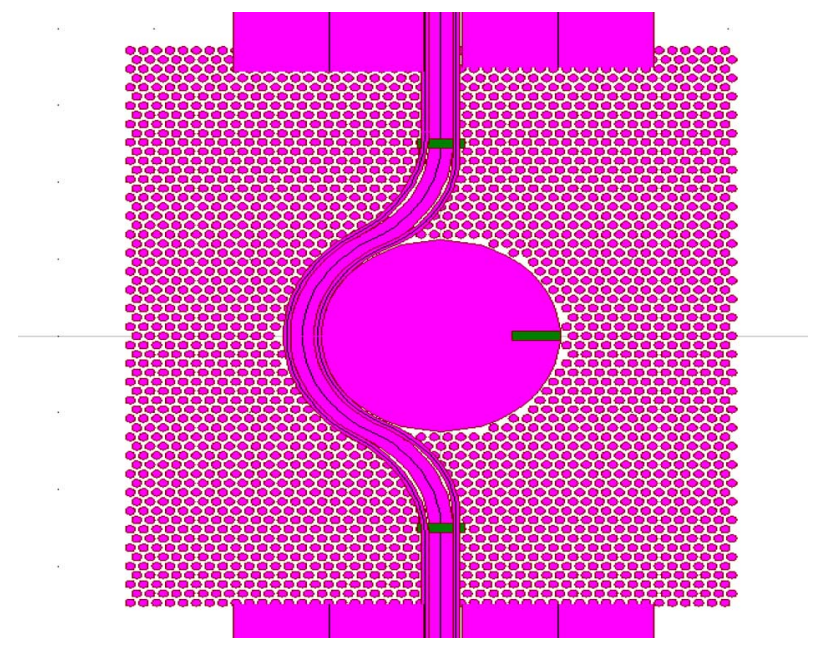

Fig. 3. Microdisk laser with PhC cladding. In this case, we introduced a small air gap (width of $150 \mathrm{~nm}$ ) between the microdisk laser and the waveguide.

$\mathrm{PhC}$ (in all directions in the plane $x-z$ ) is between $922 \mathrm{~nm}$ and $1061 \mathrm{~nm}$. In this bandgap region, light cannot propagate through the $\mathrm{PhC}$ region and is forced to funnel through the waveguide region or escape in the vertical direction.

The main magnetic field spectrum $\left(H_{\mathrm{y}}\right)$ is shown in Fig. 4(a) with the magnetic field monitor placed at the center of the upper portion of the waveguide. A bad collateral effect of the introduction of the $\mathrm{PhC}$ cladding is the appearance of many additional resonant modes due to the tighter lateral confinement (in case of an air cladding, low-quality factor modes suffered higher radiation losses). Moreover, we can no longer claim that the main peak in the region where the QWs have high gain is $\mathrm{TE}_{18,2}$. The main peak (the resonant mode with largest quality factor in the gain region of the QWs) appears at $\lambda=976 \mathrm{~nm}$ and is no longer a whispering gallery mode. The reduction in the lateral losses is strongly reflected at the $Q$ of this resonant mode, which increases to 11200 . A power budget analysis indicates that $86 \%$ is now coupled into the waveguide, the only losses being accounted for those losses in the vertical direction. This can be observed in Fig. 4(b) where we can observe that the PhC is operating in the bandgap region and there are virtually no lateral losses.

We also analyze the reduction of the air gap between the waveguide and the microdisk to a zero width. The magnetic field $\left(H_{\mathrm{y}}\right)$ spectrum is shown in Fig. 5, which is very similar to that in Fig. 4(a). The main peak appears at $\lambda=976 \mathrm{~nm}$ with quality factor of 6100. This $Q$ is higher than in the case of the structure shown in Fig. 6(b) but is considerably lower than that in the Fig. 4(a). This can be explained by an improved coupling of light into the waveguide. A power budget analysis indicates that $87 \%$ of the input power is now coupled into the waveguide. The magnetic field distribution $\left(H_{\mathrm{y}}\right)$ of this resonant peak is shown in Fig. 7. The merging of the waveguide and the microdisk laser does not result in a major improvement of the overall coupling efficiency because the loss limitation is now due to the vertical losses, since light is very tightly confined laterally.

A further improvement of coupling efficiency implies the reduction of the vertical losses. One way to reduce the vertical 


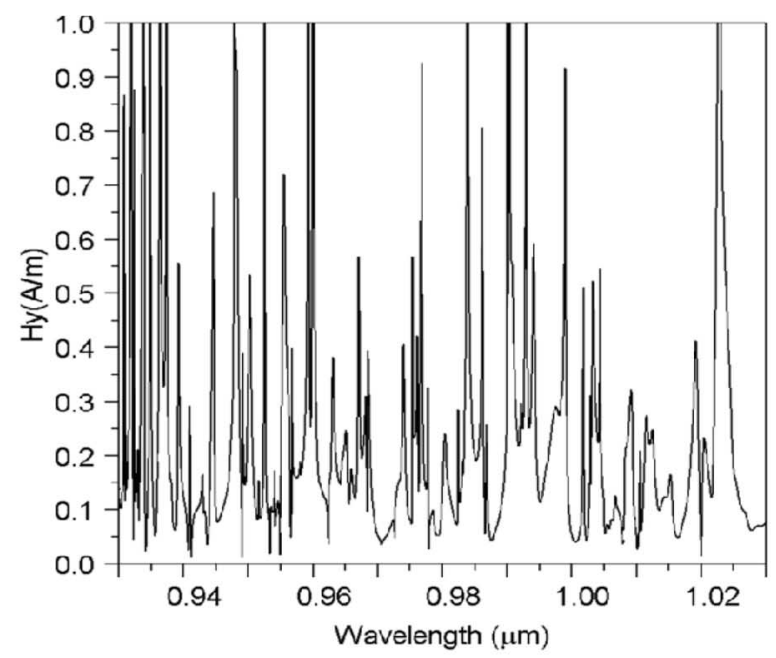

(a)

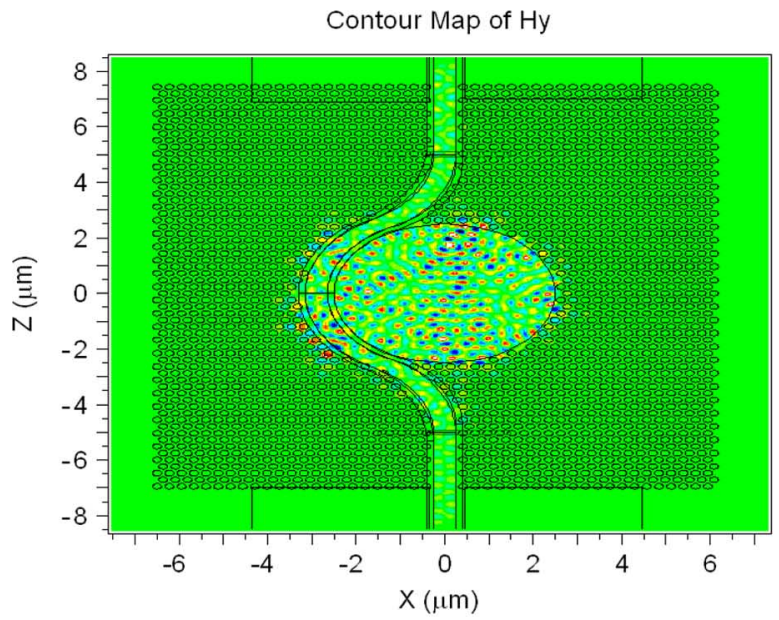

(b)

Fig. 4. (a) Magnetic field spectrum $\left(H_{\mathrm{y}}\right)$ of the microdisk coupled to a waveguide and surrounded by PhC cladding. (b) Magnetic field distribution at $\lambda=$ $976 \mathrm{~nm}$.

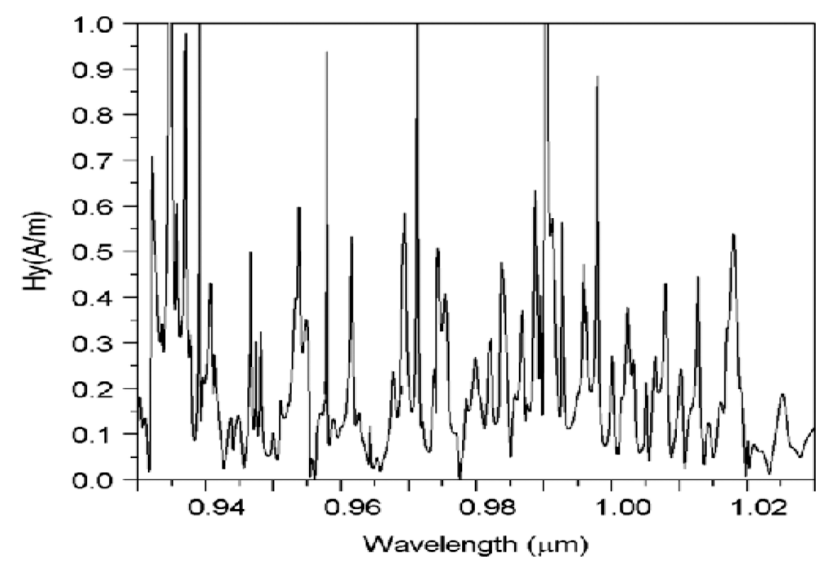

Fig. 5. Magnetic field spectrum of the microdisk overlapping with a waveguide and surrounded by PhCs.

losses is to substitute the oxide region by a Bragg stack operating in its bandgap region. Although it would be harder for light to escape through the highly reflecting bottom Bragg stack, light could still escape through the air region (besides the obvious

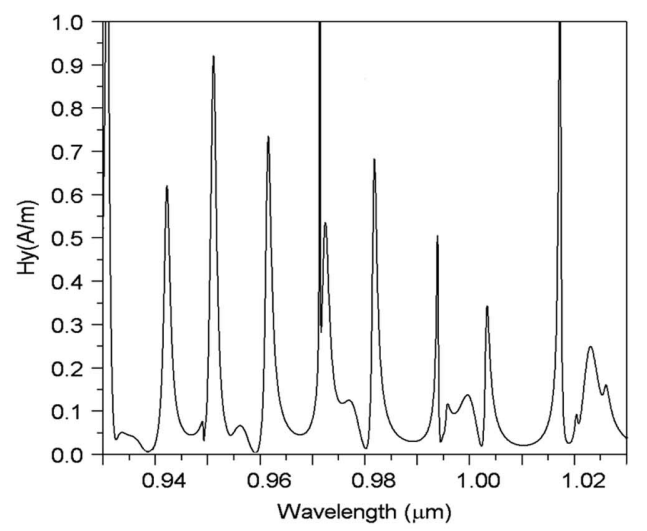

(a)

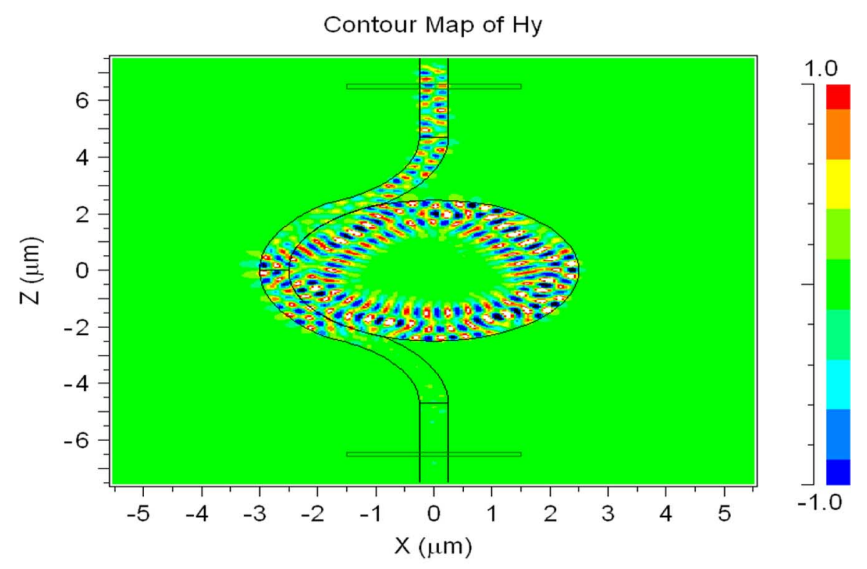

(b)

Fig. 6. (a) Magnetic field spectrum $\left(H_{\mathrm{y}}\right)$ of the merged microdisk laser and wrapped waveguide. (b) Magnetic field distribution $\left(H_{\mathrm{y}}\right)$ at $\lambda=971 \mathrm{~nm}$.

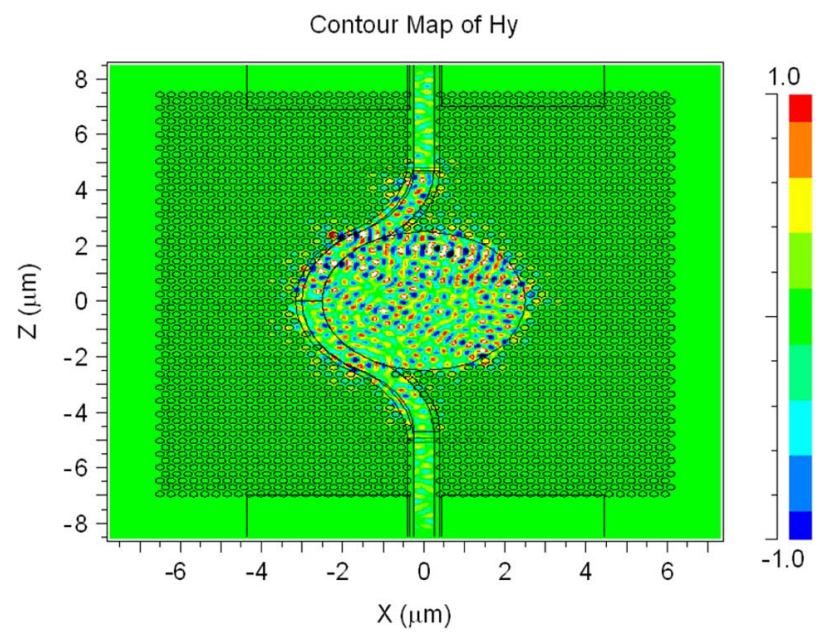

Fig. 7. Magnetic field distribution of the microdisk overlapping with a wrapped waveguide and surrounded by $\mathrm{PhCs}$ at the main peak.

route through the waveguide). In all our calculations, the power escaping through the air layer was below 5\%; hence, we could expect that more than $95 \%$ of the generated light could be funneled into the waveguide.

Although this solution may lead to nearly $100 \%$ coupling of light into the waveguide, it results in the generation of rather strong additional resonant peaks. This can lead to a stronger mode competition in the laser device, where the pump power 


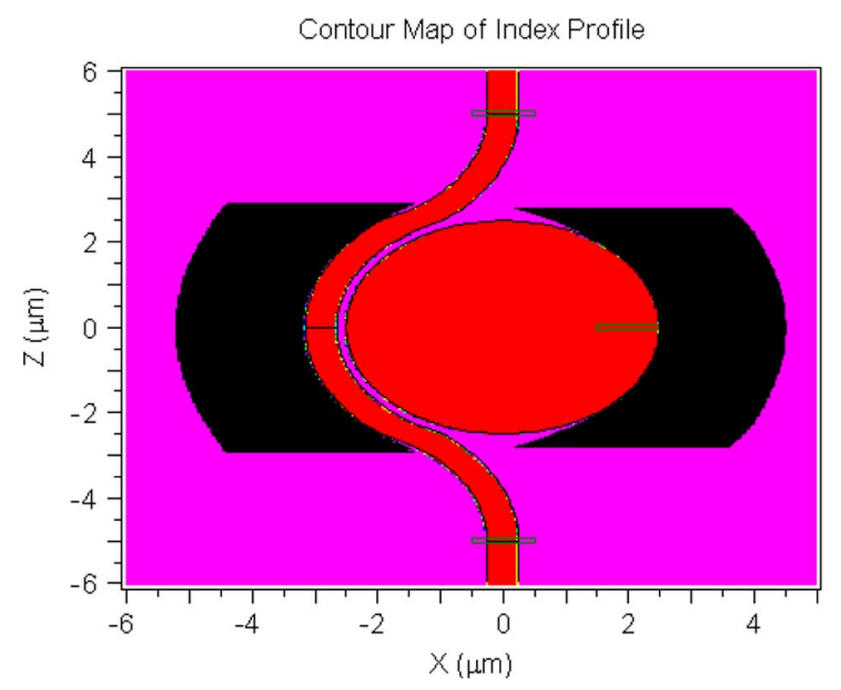

Fig. 8. Microdisk laser coupled to a waveguide with a plasmonic cladding.

will be distributed among different modes in a tentative to reach lasing. The overall consequence is an increase of the threshold power of the structure, an undesired effect.

\section{Coupling of Light Assisted By a PLASMONIC CLADDING}

Recently, it has been shown that light could be transmitted through very tiny holes, in defiance to diffraction [21]. This physical phenomenon was called "extraordinary" transmission of light. It is argued that the excitation of surface plasmons can funnel light into these subwavelength apertures, what is not normally possible with dielectric structures. This phenomenon appears very promising to the development of very compact structures such as filters, waveguides, and sensors.

The discovery of the extraordinary transmission of light has opened up the possibility of confining light in very small spaces, even further than with low refractive indexes (with total internal reflection) and with $\mathrm{PhC}$ claddings. However, one major obstacle to fully using plasmonic cladding is the metal losses. Another special feature of plasmonic devices is the generation of high intense electric fields, which can be used in biological and chemical sensing applications [23], [24].

In order to assess the efficiency of a plasmonic cladding, we substitute the classical air cladding by a plasmonic cladding. This plasmonic cladding consists of lateral layers of gold as shown in Fig. 8 (black regions in this figure). There are not as many peaks as in Fig. 2(b), as can be observed in the $H_{\mathrm{y}}$ spectrum in this figure. However, the main peak appears at $\lambda=$ $984.35 \mathrm{~nm}$ with $Q=1500$ [see Fig. 9(a)]. This quality factor is considerably lower than in the previous cases, an indication that the metal losses play a major role in the performance of the device.

The field distribution at $\lambda=984.35 \mathrm{~nm}$ is shown in Fig. 9(b). However, a power budget analysis indicates that only $40 \%$ of the generated power is coupled into the waveguide, $13 \%$ is lost in the vertical direction while $47 \%$ is lost laterally and is quickly dissipated in the metallic region.

In the previous approach, we tried to introduce the plasmonic regions as reflectors around the waveguide and microdisk res-

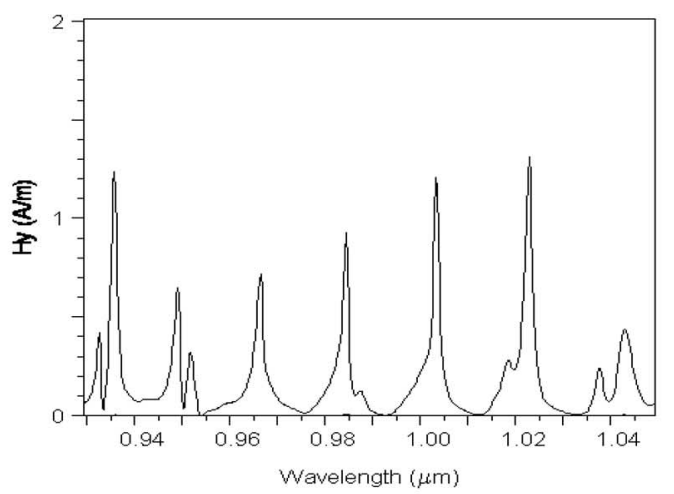

(a)

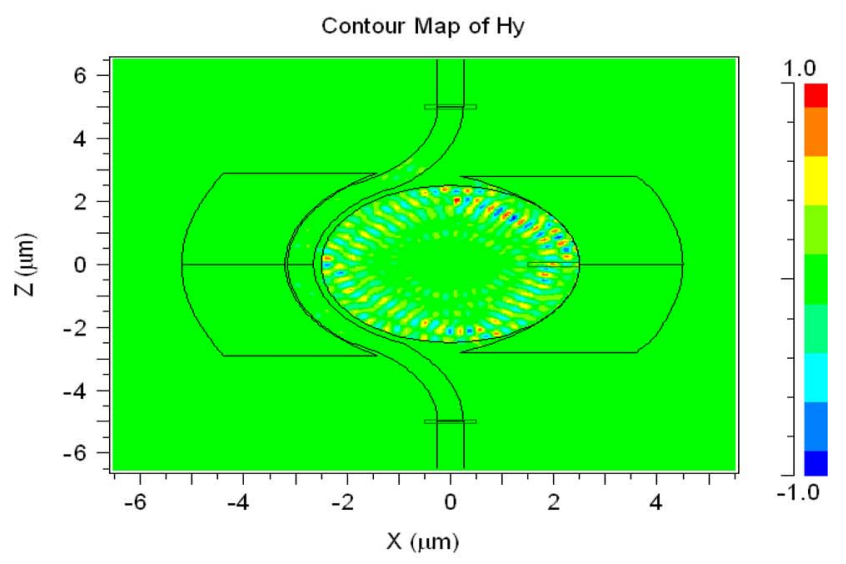

(b)

Fig. 9. Microdisk laser coupled to a waveguide with a plasmonic cladding. (a) Magnetic field $\left(H_{\mathrm{y}}\right)$ spectrum at the center of the waveguide and (b) field distribution at $\lambda=978 \mathrm{~nm}$.

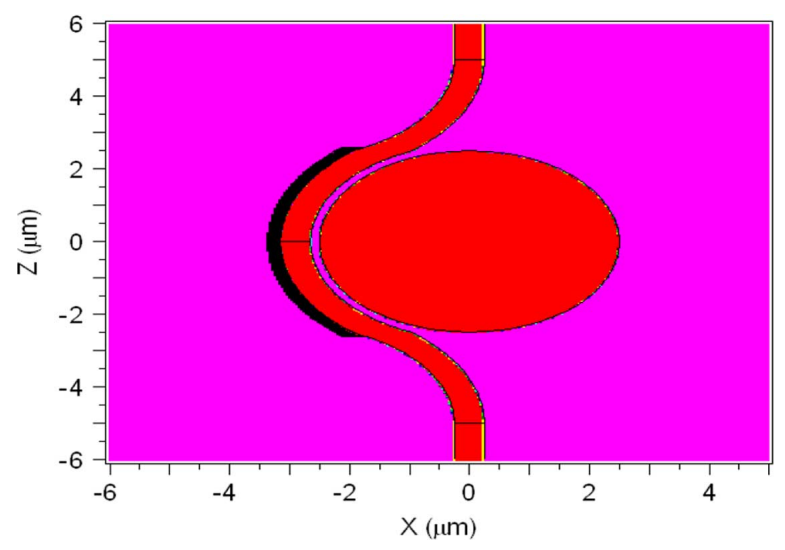

Fig. 10. Microdisk laser coupled to a waveguide with a plasmonic cladding beside the waveguide.

onator. This approach proved inefficient because of the metal losses and poor shielding provided by the plasmonic layers. However, a better approach is obtained by placing a plasmonic layer beside the waveguide, as shown in Fig. 10.

We changed the thickness of the metallic layer (dark region) and assessed the quality factor of the main mode (peak with the highest quality factor in the gain region of the $\mathrm{QW}$ ) as a function of the thickness of the cladding layer. The results are shown in Fig. 11. The increase of the gold layer leads to an increase in the absorption of light in the metallic regions but can also lead 


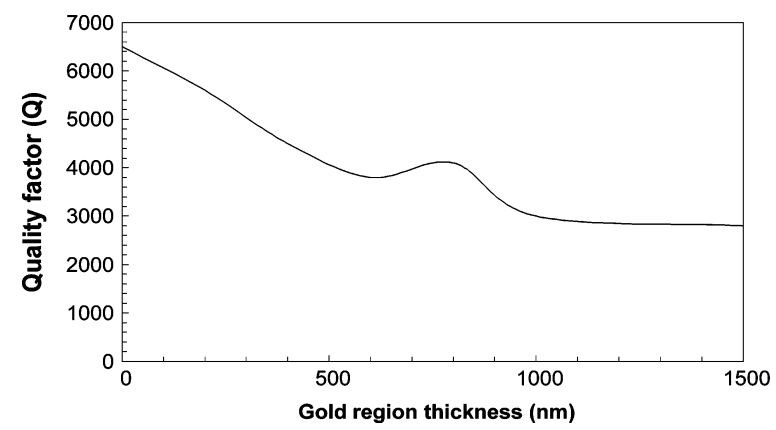

Fig. 11. Microdisk laser coupled to a waveguide with a plasmonic cladding beside the waveguide. Quality factor as a function of the gold thickness.

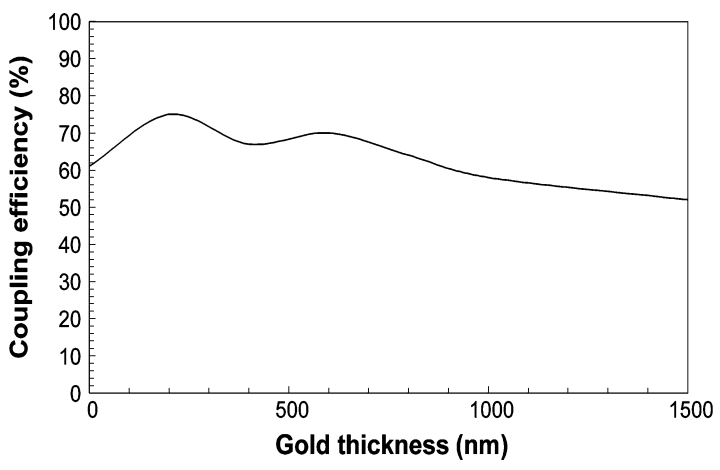

Fig. 12. Microdisk laser coupled to a waveguide with a plasmonic cladding beside the waveguide. Coupling efficiency as a function of the gold thickness.

to a reduction in the radiation losses of the structure. This may explain a small oscillation at a thickness of $800 \mathrm{~nm}$. However, the general trend is that an increase in the thickness of the gold layer leads to a reduction of the quality factor of the main mode that appears at $\lambda=972.62 \mathrm{~nm}$ and closely resembles the mode $\mathrm{TE}_{18,2}$. Moreover, the $H_{\mathrm{y}}$ spectrum is similar to the one presented in Fig. 2(b), with no additional peaks but lower quality factors.

A much more interesting result is obtained when we examine the effect of this one-sided plasmonic layer on the coupling efficiency as a function of the thickness of the gold layer. The results are shown in Fig. 12. Several physical mechanisms interplay in this case: absorption of light in the gold layer, reflection of light by the plasmonic layer that is funneled into the waveguide, and reflection of light by the plasmonic layer that is radiated elsewhere. The first and last physical mechanisms tend to reduce the light coupling into the waveguide, but the second physical mechanism increases the light coupling into the waveguide. This can be clearly observed for thicknesses of $200 \mathrm{~nm}$ and $800 \mathrm{~nm}$, where we can couple more light in the waveguide than in the case where the plasmonic layer was absent. In fact, the introduction of a gold layer of thickness of $200 \mathrm{~nm}$ leads to a coupling efficiency of $76 \%$, which is nearly $16 \%$ better than in the case of Fig. 2(a) (in the absence of the plasmonic layer).

The field distribution for the plasmonic-assisted coupling is shown in Fig. 13. We can see that a significant portion of the input power is indeed coupled into the waveguide. However, there is still some portion of the generated power that is radiated away from the waveguide because of the bends. The asymmetric

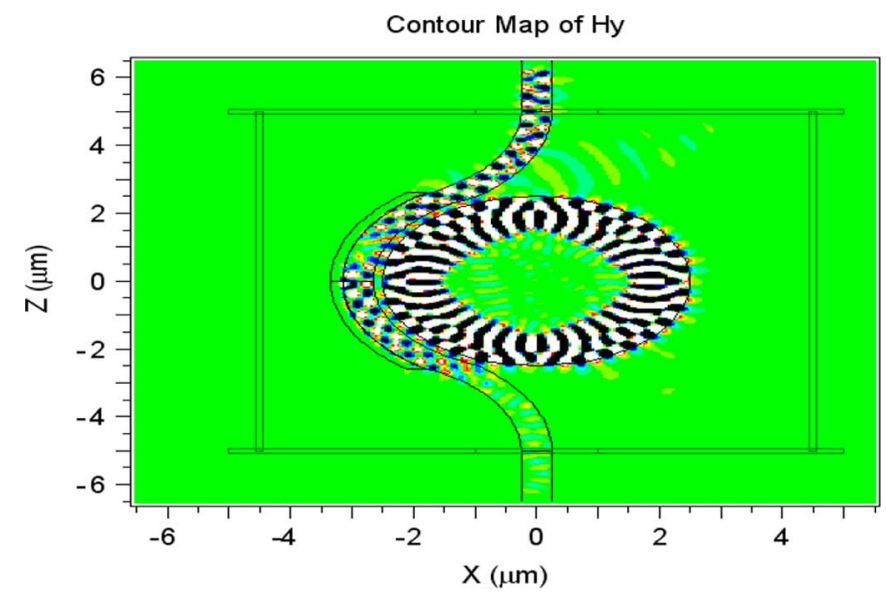

Fig. 13. Electromagnetic filed distribution for a microdisk laser coupled to a waveguide with a plasmonic cladding beside the waveguide.

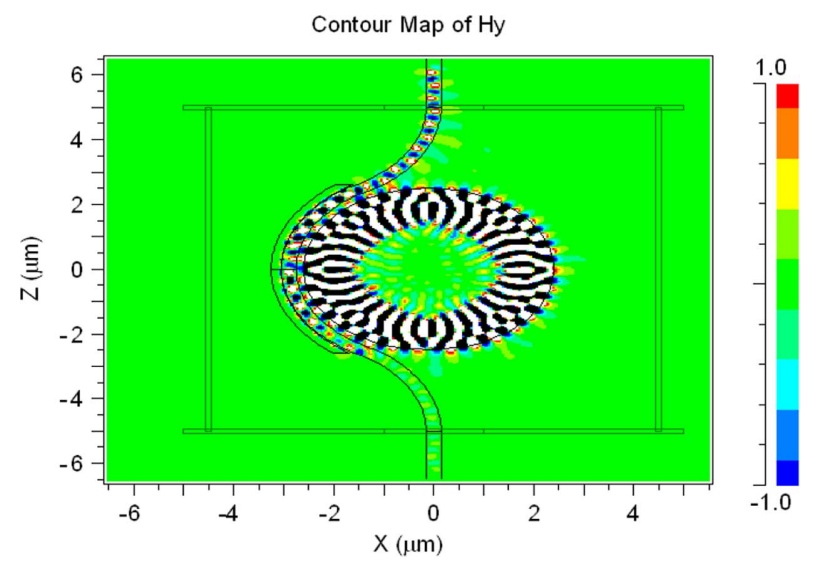

Fig. 14. Electromagnetic filed distribution for a microdisk laser coupled to a $300 \mathrm{~nm}$ wide single-mode waveguide with a plasmonic cladding beside the waveguide.

coupling of light is an artifact of our simulation: the source is generating light that propagates clockwise with the result of "artificially" coupling more light in the $z>0$ direction).

It seems that the optimized value of the thickness of the gold layer is $200 \mathrm{~nm}$ : the quality factor is not so low $(Q=5600$, since there is not much absorption by the plasmonic mirror) and the plasmonic layer helps to funnel light into the waveguide. In fact, a closer look at the interface between the metallic and waveguide region shows the generation of high intense local electric fields, showing the excitation of plasmonic waves. With further improvement in the confinement in the vertical direction (e.g., introduction of a bottom Bragg stack), it would be possible to reach even higher values of coupling efficiency. In the absence of vertical losses, the coupling efficiency could reach values close to $90 \%$.

Now, if we reduce the width of the waveguide to $300 \mathrm{~nm}$ (truly single-mode as seen in Fig. 14), the coupling efficiency is reduced to about $71 \%$. In the absence of vertical losses, the coupling efficiency would reach values of about $81 \%$. The increase of the waveguide width to $500 \mathrm{~nm}$ leads to more light being coupled into the waveguide, but at the expense of exciting higher order modes. 


\section{Steady-State Laser Analysis of a Few SELECTED CASES}

Equations (2) and (3) [11], [32] represent the rate equations for carrier and photon densities. These devices are going to be pumped optically in the vertical direction by an external optical pumping laser with a spot diameter of $6 \mu \mathrm{m}$. These equations are able to provide an estimate for the laser performance of these combined microdisk and waveguide structures.

$$
\begin{aligned}
\frac{d N}{d t} & =\eta \frac{P_{\mathrm{in}} \lambda_{\mathrm{p}}}{h c_{\mathrm{o}} V_{\mathrm{a}}}-\left(A N+B N^{2}+C N^{3}\right)-\Gamma G(N) S \\
\frac{d S}{d t} & =\Gamma G(N) S+\beta B N^{2}-\frac{S}{\tau_{\mathrm{p}}}
\end{aligned}
$$

where $N$ and $S$ are the carrier and photon densities, respectively, $P_{\text {in }}$ is the optical pump power, $c_{\mathrm{O}}$ is the free-space velocity of light, $\eta$ is the absorption ratio of the pump in the QW region, $\lambda_{\mathrm{p}}$ is the pump wavelength, $A, B$, and $C$ are recombination coefficients, $\Gamma$ is the confinement factor, $G(N)$ the gain of the QWs, $\beta$ is the spontaneous emission factor, $\tau_{\mathrm{p}}$ is the photon lifetime, and $V_{\mathrm{a}}$ is the active pump volume. The active pump volume $V_{\mathrm{a}}$ is given by

$$
V_{a}=h_{\mathrm{a}} \pi \frac{\phi_{\mathrm{s}}^{2}}{4}
$$

where $h_{\mathrm{a}}$ is the thickness of the active region and $\phi_{\mathrm{s}}$ is the spot size diameter of the external pump laser.

The time constant $\tau_{\mathrm{p}}$ is given by [32]

$$
\tau_{\mathrm{p}}=\frac{Q \lambda_{e}}{2 \pi c_{o}}
$$

where $\lambda_{e}$ the emitting wavelength and $Q$ is the quality factor of the resonant mode.

The gain $G(N)$ of the QWs can be expressed as [32]

$$
G(N)=v_{\mathrm{g}} G_{\mathrm{o}} \ln \left(\frac{N}{N_{\mathrm{tr}}}\right)
$$

where $v_{\mathrm{g}}$ is the group velocity of the mode, $G_{\mathrm{o}}$ is the gain coefficient (typical value of $1.5 \times 10^{5} \mathrm{~m}^{-1}$ ), and $N_{\mathrm{tr}}$ is the transparency carrier density with a typical value of $1.5 \times 10^{24} \mathrm{~m}^{-3}$.

In the steady-state regime, the two derivatives in (2) and (3) are equal to zero, allowing us to determine the steady-state photon and carrier densities. Once we determine the steady-state photon density $S$, we can find the output power by using (7) [32]

$$
P_{\text {out }}=\eta_{a} \frac{h c_{o}}{\lambda_{e}} S \frac{V_{\text {mode }}}{\tau_{\text {mirror }}}
$$

where $\eta_{\mathrm{a}}$ is the coupling efficiency of the waveguide, $\tau_{\text {mirror }}$ is the mirror lifetime, which is assumed to have the same value as $\tau_{\mathrm{p}}$ [32], and $V_{\text {mode }}$ is the optical mode volume which expressed

\begin{tabular}{|c|c|c|}
\hline Symbol & Quantity & Typical value \\
\hline$\eta$ & $\begin{array}{l}\text { Absorption ratio of } \\
\text { pump in } Q W \text { region }\end{array}$ & 0.26 \\
\hline$\lambda_{\mathrm{p}}$ & Pump wavelength & $637 \mathrm{~nm}$ \\
\hline $\mathrm{h}$ & Planck's constant & $6.626 \times 10^{-34} \mathrm{~kg} / \mathrm{s}$ \\
\hline$c_{\mathrm{o}}$ & $\begin{array}{c}\text { Free-space velocity of } \\
\text { light }\end{array}$ & $299792458 \mathrm{~m} / \mathrm{s}$ \\
\hline$\eta_{\mathrm{a}}$ & $\begin{array}{l}\text { Coupling efficiency to } \\
\text { the waveguide }\end{array}$ & 0.7 \\
\hline A & $\begin{array}{l}\text { Linear recombination } \\
\text { coefficient }\end{array}$ & $2.5 \times 10^{8} \mathrm{~s}^{-1}$ \\
\hline B & $\begin{array}{c}\text { Bimolecular } \\
\text { recombination } \\
\text { coefficient }\end{array}$ & $1.6 \times 10^{-16} \mathrm{~m} / \mathrm{s}$ \\
\hline$C$ & $\begin{array}{l}\text { Auger non-radiative } \\
\text { recombination rate }\end{array}$ & $5 \times 10^{-41} \mathrm{~m}^{6} / \mathrm{s}$ \\
\hline$\Gamma$ & Confinement factor & 0.22 \\
\hline$\beta$ & $\begin{array}{l}\text { Spontaneous emission } \\
\text { factor }\end{array}$ & $10^{-41}$ \\
\hline
\end{tabular}
as

$$
V_{\text {mode }}=\mathrm{h}_{\mathrm{p}} \pi \mathrm{r}^{2}
$$

TABLE I

TYPICALLASER PARAMETERS

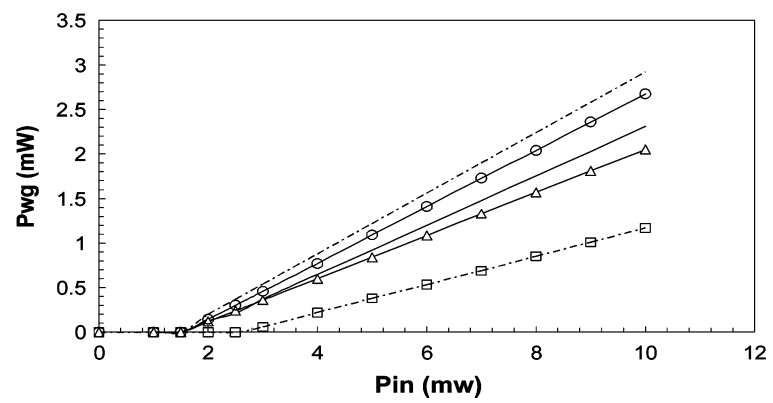

Fig. 15. Steady-state response for the microdisk coupling in five different cases: dotted line with no markers for the PhC cladding, solid line with circular markers for the one-sided plasmonic cladding beside the waveguide, solid line with no markers for the merged waveguide-microdisk structure, solid line with triangular markers for the microdisk with a wrapped waveguide separated by an air gap and dotted curve with square markers for a double plasmonic cladding.

where $h_{\mathrm{p}}$ is the thickness of the active layer and $r$ is the radius of the microdisk resonator. Typical values of the remaining parameters are summarized in Table I.

This laser structure can lase at distinct resonant wavelengths. However, the mode with highest $Q$ will be the first mode to lase and we will present some steady-state laser responses for this main mode. We will analyze the amount of power coupled into the waveguide $\left(P_{\mathrm{wg}}\right)$. The cases under consideration are shown in Fig. 15: (a) microdisk with a wrapped waveguide and an air gap (solid curve with triangular markers), (b) microdisk with overlapping wrapped waveguide (solid curve), (c) microdisk with double plasmonic cladding (dotted curve with square markers), and (d) microdisk with a single plasmonic cladding beside the waveguide (solid curve with circular markers). For sake of comparison only, we add an additional case (case (e), dotted curve) in which we add the PhC cladding (with the warning that the "generation" of many additional modes may lead to strong mode competition and lead to a much higher threshold level than the one presented in the plot). It is clear 
from these curves that the plasmonic-assisted coupling (a single metallic layer beside the waveguide) has improved the coupling of light into the waveguide without an acute reduction of the quality factor of the combined structure (which would lead to a much higher threshold pump power) and this curve approaches the curve in which there would not be any lateral losses (dotted curve).

\section{CONCLUSION}

We analyzed the coupling efficiency of a microdisk laser and a single-mode waveguide with three different claddings: air, $\mathrm{PhC}$, and plasmonic claddings. The photonic crystal cladding provides the best coupling efficiency into the waveguide but at the expense of generating many additional resonant peaks, which may lead to strong mode competition in the laser device. The introduction of a single metallic layer funneled light into the waveguide leading to a significant improvement in the coupling efficiency without the generation of many additional modes and seems to be the best coupling scheme studied in this article.

\section{REFERENCES}

[1] H. G. Park, J. K. Hwang, J. Huh, H. Y. Ryu, S. H. Kim, J. S. Kim, and Y. H. Lee, "Characteristics of modified single-defect two-dimensional photonic crystal lasers," IEEE J. Quantum Electron., vol. 38, no. 10, pp. 1353-1365, Oct. 2002.

[2] H. T. Hattori, I. McKerracher, H. H. Tan, C. Jagadish, and R. M. De La Rue, "In-plane coupling of light from InP-based photonic crystal bandedge lasers into single-mode waveguides," IEEE J. Quantum Electron., vol. 43 , no. 3, pp. 279-286, Mar. 2007.

[3] R. M. Cazo, C. L. Barbosa, H. T. Hattori, and V. M. Schneider, "Steady-state analysis of a directional square lattice band-edge photonic crystal laser," Microw. Opt. Technol. Lett., vol. 46, no. 3, pp. 210-214, 2005.

[4] H. T. Hattori, H. H. Tan, and C. Jagadish, "Optically pumped in-plane photonic crystal micro-cavity laser arrays coupled to waveguides," $J$. Lightw. Technol., vol. 26, no. 11, pp. 1374-1380, Jun. 1, 2008.

[5] S. Ando, N. Kobayashi, and H. Ando, "Triangular-facet laser with optical waveguides grown by selective area metalorganic chemical vapor deposition," Jpn. J. Appl. Phys., vol. 35, no. 4a, pt. 2, pp. L411-L413, 1996.

[6] S. Ando, N. Kobayashi, and H. Ando, "Triangular-facet lasers coupled by a rectangular optical waveguide," Jpn. J. Appl. Phys., vol. 36, no. 2a, pt. 2, pp. L76-L78, 1997.

[7] Y. Z. Huang, W. H. Guo, and Q. M. Wang, "Analysis and numerical simulation of eigenmode characteristics for semiconductor lasers with an equilateral triangle micro-resonator," IEEE J. Quantum Electron., vol. 37, no. 1, pp. 100-107, Jan. 2001.

[8] H. T. Hattori, "Analysis of optically pumped equilateral triangular microlasers with three mode-selective trenches," Appl. Opt., vol. 47, no. 12, pp. 2178-2185, 2008.

[9] W. H. Guo, Y. Z. Huang, Q. Y. Lu, and L. J. Yu, "Mode quality factor based on far-field emission for square resonators," IEEE Photon. Technol. Lett., vol. 16, no. 2, pp. 479-481, Feb. 2004.

[10] S. V. Boriskina, T. M. Benson, P. Sewell, and A. I. Nosich, "Spectral shift and Q-change of circular and square-shaped optical micro-cavity modes due to periodic sidewall surface roughness," J. Opt. Soc. Amer. $B$, vol. 21, no. 10, pp. 1792-179, 2004.

[11] H. T. Hattori, "Modal analysis of one-dimensional non-uniform arrays of square resonators," J. Opt. Soc. Amer. B, vol. 25, no. 11, pp. 1873-1881, 2008.

[12] Y. Z. Huang, K. J. Che, Y. D. Yang, S. J. Wang, Y. Du, and Z. C. Fan, "Directional emission InP/GaInAsP square-resonator microlasers," Opt. Lett., vol. 33, no. 19, pp. 2170-2172, 2008.

[13] H. T. Hattori, D. Liu, H. H. Tan, and C. Jagadish, "Large square resonator laser with quasi-single-mode operation," IEEE Photon. Technol. Lett., vol. 21, no. 6, pp. 359-361, Mar. 15, 2009.
[14] M. Fujita, A. Sakai, and T. Baba, "Ultra-small and ultra-low threshold microdisk injection laser-design, fabrication, lasing characteristics and spontaneous emission factor," IEEE J. Sel. Topics Quantum Electron., vol. 5, no. 3, pp. 673-681, May/Jun. 1999.

[15] A. F. J. Levi, R. E. Slusher, S. L. McCall, J. L. Glass, S. J. Pearton, and R. A. Logan, "Directional light coupling from microdisk lasers," Appl. Phys. Lett., vol. 62, no. 6, pp. 561-563, 1993.

[16] S. J. Choi, K. Djordjev, and P. D. Dapkus, "Microdisk lasers vertically coupled to output waveguides," IEEE Photon. Technol. Lett., vol. 15, no. 10 , pp. 1330-1332, Oct. 2003.

[17] H. T. Hattori, C. Seassal, E. Touraille, P. Rojo-Romeo, X. Letartre, G. Hollinger, P. Viktorovitch, L. DiCioccio, M. Zussy, L. El Melhaoui, and J. M. Fedeli, "Heterogeneous integration of microdisk lasers on silicon strip waveguides for optical interconnects," IEEE Photon. Technol. Lett., vol. 1, no. 1, pp. 223-225, Jan. 2006.

[18] X. Wu, H. Li, L. Liu, and L. Xu, "Unidirectional single-frequency lasing from a ring-spiral coupled microcavity laser," Appl. Phys. Lett., vol. 93, no. 8, pp. 081105-1-081105-3, 2008, article no. 081105.

[19] X. Luo and A. W. Poon, "Coupled spiral-shaped microdisk resonators with non evanescent asymmetric inter-cavity coupling," Opt. Exp., vol. 15, no. 25, pp. 17313-17322, 2007.

[20] Y. Z. Huang and Y. D. Yang, "Mode coupling and vertical radiation loss for whispering-gallery modes in 3-D microcavities," J. Lightw. Technol., vol. 26, no. 11, pp. 1411-1416, Jun. 1, 2008.

[21] C. Genet and T. W. Ebbesen, "Light in tiny holes," Nature, vol. 445, no. 7123, pp. 39-46, 2007.

[22] E. Laux, C. Genet, T. Skauli, and T. W. Ebbesen, "Plasmonic photon sorters for spectral and polarimetric imaging," Nat. Photon., vol. 2, no. 3, pp. 161-164, 2008.

[23] K. Leosson, T. Nikolajsen, A. Boltasseva, and S. I. Bozhevolnyi, "Long range surface plasmon polariton nanowire waveguides for device applications," Opt. Exp., vol. 14, no. 1, pp. 314-319, 2006.

[24] J. A. Porto, L. M. Moreno, and F. J. G. Vidal, "Optical bistability in sub-wavelength slit apertures containing nonlinear media," Phys. Rev. $B$, vol. 70, no. 8, pp. 081402-1-081402-4, 2004, article no. 081402.

[25] C. Y. Fong and A. W. Poon, "Planar corner-cut square microcavities: Ray optics and FDTD analysis," Opt. Exp., vol. 12, no. 20, pp. 4864-4874, 2004.

[26] S. C. Hagness, D. Hafizadeh, S. T. Ho, and A. Taflove, "FDTD microcavity simulations: Design and experimental realization of waveguide-coupled single-mode ring and whispering-gallery-mode disk resonators," J. Lightw. Technol., vol. 15, no. 11, pp. 2154-2165, Nov. 1997.

[27] S. V. Boriskina, T. M. Benson, P. D. Sewell, and A. I. Nosich, "Directional emission, increased free spectral range, and mode Q-factors in 2-D wavelength-scale optical microcavity structures," IEEE J. Sel. Topics Quantum Electron., vol. 12, no. 6, pp. 1175-1182, Nov./Dec. 2006.

[28] V. Van, P. P. Absil, J. V. Hryniewicz, and P. T. Ho, "Propagation loss in single-mode GaAs-AlGaAs microring resonators: Measurement and model," J. Lightw. Technol., vol. 19, no. 11, pp. 1734-1739, Nov. 2001.

[29] J. P. Zhang, D. Y. Chu, S. L. Wu, W. G. Bi, R. C. Tiberio, C. W. Tu, and S. T. Ho, "Directional light output from photonic-wire microcavity semiconductor lasers," IEEE Phot. Technol. Lett., vol. 8, no. 8, pp. 968-970, Aug. 1996.

[30] B. Cagnac and J. P. Faroux, Lasers: Interaction Lumiere-Atomes, 1st ed. Paris: EDP Sciences et CNRS ed., 2002.

[31] H. T. Hattori, V. M. Schneider, R. M. Cazo, and C. L. Barbosa, "Analysis of strategies to improve the directionality of square lattice band-edge photonic crystal structures," Appl. Opt., vol. 44, no. 15, pp. 3069-3076, 20, 2005.

[32] H. Altug and J. Vuckovic, "Photonic crystal nanocavity array laser," Opt. Exp., vol. 13, pp. 8819-8828, 2005.

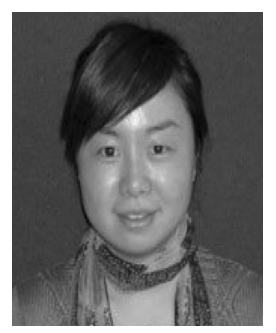

Danyu Liu (S'08) was born in China, in 1983. She received the B.Tech. degree in optoelectronics from The University of Auckland, Auckland, New Zeland, in 2006. Currently, she is working toward the Ph.D. degree in Prof. Jagadish's group in Electronic Materials Engineering, Research School of Physics and Engineering, The Australian National University, Canberra, Australia, since June 2007.

Her recent research interests include design and fabrication of 2-D and 3-D photonic crystal lasers and plasmonic devices. 


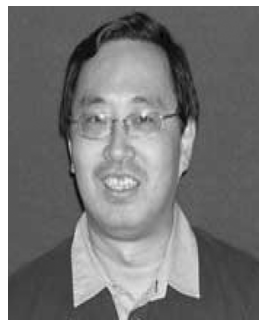

Haroldo T. Hattori (M'00-SM'08) received the B.Sc. degree (with honors) from Instituto Tecnologico de Aeronáutica (ITA), Sao Jose dos Campos, Brazil, in 1988, and the Ph.D. degree from Virginia Polytechnic Institute and State University, Blacksburg, in 1998, both in electrical engineering.

He worked for Alcatel (in Brazil and Spain) as an Optical Fiber Communications Engineer from 1989 to 1994. In 1994, he left Alcatel to work toward his Ph.D. degree at Virginia Tech. His thesis analyzed the detrimental effects of nonlinearities and dispersion for very long distance optical fiber links. In 1998, he went back to Brazil, where he became an Assistant Professor at ITA and worked on the development of fiber Bragg gratings sensors and communication devices. From 2002 to 2005, he worked as a Postdoctoral Researcher at the University of Glasgow, Glasgow, U.K., and Ecole Centrale de Lyon, Lyon, France, working with integrated optical components (photonic crystal passive and active devices and microdisk lasers). In Europe, he also worked in the integration of III-V semiconductor lasers and silicon passive devices (PICMOS project). In 2006, he moved to Australia, where he is currently a Lecturer at the School of Engineering and Information Technology at the University of New South Wales at ADFA. He is also a Visiting Fellow at the Australian National University. His research interests include semiconductor-based integrated optics (mostly active devices), plasmonics, meta-materials, nanotechnology, optical communications, biological sensors, quantum heterostructures, optical fibers, bioinformatics, and computers. He has authored or coauthored more than 40 journal papers and more than 60 conference papers.

Dr. Hattori is a Senior Member of the Institute of Electrical and Electronic Engineers (IEEE), Senior Member of IEEE-LEOS, Member of the Optical Society of America (OSA), Member of the Institution of Engineers Australia, Member of Phi Kappa Phi and Eta Kappa Nu Honor Societies.

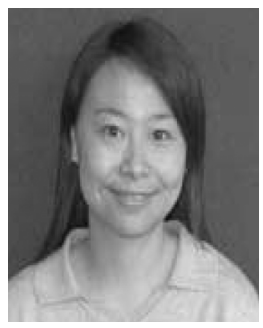

Lan Fu (S'99-M'01-SM'07) received the B.E. degree from the Hefei University of Technology, Hefei, China, in 1993, and M.S. degree from the University of Science and Technology of China, Hefei, in 1996, and the Ph.D. degree from The Australia National University, Canberra, Australia, in 2001, for work on quantum-well intermixing in (In)GaAs/GaAs materials and devices.

She is currently a Fellow in the Department of Electronic Materials Engineering, The Australian National University. Her research interests include MOCVD growth of III-V semiconductor materials, design, fabrication, and integration of optoelectronic devices.

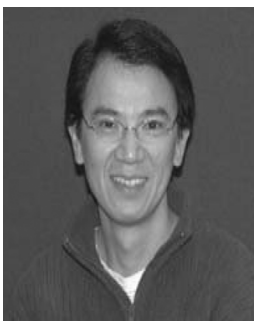

Hoe H. Tan (S'93-M'96-SM'03) received the B.E. (Elec.) and Ph.D. degrees from the University of Melbourne, Victoria, Australia, and the Australian National University, Canberra, Australia, in 1992 and 1997, respectively.

In 1992, he joined Siemens Opto-Semiconductor, Penang, where he worked in the area of quality assurance. His doctoral dissertation was on ion beam effects in GaAs-AlGaAs materials and devices. His research interests include fundamental and applied aspects of compound semiconductors. He has authored and coauthored more than 180 journal papers. The two main contributions of his work to the field are in MOCVD growth of nanostructures and ion implantation of semiconductors for optoelectronic applications. He is currently a Senior Fellow at the Australian National University.

Dr. Tan was a former recipient of the prestigious Australian Research Council Queen Elizabeth II fellowship.

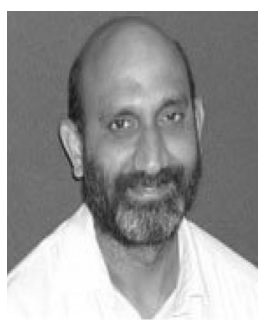

Chennupati Jagadish (S'84-M'89-SM'92-F'02) received the B.Sc., M.Sc.(Tech.), M.Phil., Ph.D. degrees in India.

He moved to Australia in 1990 and established a major research program in the field of semiconductor optoelectronics and nanotechnology. He is currently a Federation Fellow, Distinguished Professor and Head of Semiconductor Optoelectronics and Nanotechnology Group in the Department of Electronic Materials Engineering, Research School of Physics and Engineering, the Australian National University (ANU). He is also convener of the Australain Research Council Nanotechnology Network and Director (ACT Node) of the Australian National Fabrication Facility. Currently, he is serving as President of the IEEE Nanotechnology Council from 2008 to 2009, and served as Vice-President (Membership and Regional Activities-Asia-Pacific) of IEEE LEOS from 2006 to 2008, and also served as a member of IEEE Fellow Selection Committee from 2006 to 2008. His research interests include compound semiconductor optoelectronics and nanotechnology including quantum dots, nanowires, lasers, photodetectors, photonic integrated circuits, photonic crystals, and $\mathrm{THz}$ photonics. He has authored and coauthored more than 550 research papers ( 370 journal papers), holds five US patents, co-authored a book, co-edited a book and edited ten conference proceedings, guest edited five journal special issues.

Dr. Jagadish is a winner of 2000 IEEE Millennium Medal, Peter Baume Award from the ANU and served as a Distinguished Lecturer of both IEEE Lasers and Electro-Optics Society (LEOS) and IEEE Electron Devices Society (EDS). He is a Fellow of the IEEE, APS, OSA, AVS, SPIE, ECS, AAAS, IET, AIP, IoP, IoN, the Australian Academy of Technological Sciences and Engineering and the Australian Academy of Science. He also served as Vice-President (Publications) of the IEEE Nanotechnology Council. 